\title{
On the Environmental Dependence of Galaxy Properties Established by the Initial Cosmological Conditions
}

\author{
Jounghun LeE \\ Department of Physics and Astronomy, Seoul National University, Seoul 151-742, Korea \\ jounghun@astro.snu.ac.kr
}

\begin{abstract}
We study theoretically how the initial cosmological conditions establish the dependence of galaxy properties on the environment. First, we adopt the linear tidal torque theory according to which the angular momentum of a proto-galaxy is generated at first order by the misalignment between the proto-galaxy inertia tensor and the local tidal tensor. Then, we quantify analytically the degree of the misalignment between the two tensors, and show quantitatively that it increases as the density of the environment decreases. It implies that the protogalaxies forming in the lower density regions should end up with having higher angular momentum than those in the higher density regions, which is consistent with recent numerical finding that the void and field galaxies have higher spin parameters than the cluster galaxies. Since the galaxy angular momentum plays a role of developing a disk-like structure and hindering the star-formation, our theoretical insight provides an answer to such fundamental observational question as why the large void galaxies have young stellar populations and high specific star formation rate, which was not explained by the previous morphology-density relation.
\end{abstract}

Subject headings: cosmology:theory — large-scale structure of universe

\section{INTRODUCTION}

Recent observations from the Sloan Digital Sky Survey (SDSS, Strauss et al. 2002) and the 2dF Galaxy Redshift Survey (2dFGR, Colless et al. 2001) have made it possible to study the properties of galaxies from largest spectroscopic samples. Although it has been noted for long that the morphology and the specific star formation rate of galaxies depend on the density of environments (Dressler 1980; Postman \& Geller 1984; Whitmore et al. 1993), it 
is only in recent years that the environmental dependence of galaxy properties have been observationally confirmed as common and studied systematically through statistical analyses of these large samples (Lewis et al. 2002; Gomez et al. 2003; Goto et al. 2003; Tanaka et al. 2004; Rojas et al. 2005).

Now that the environmental dependence of galaxy properties holds a crucial key to the galaxy formation, it is quite important and necessary to understand what physical mechanism establishes it. A fundamental question that naturally arise in the study of the environmental dependence of galaxy properties is whether it is destined from the initial conditions or acquired during the evolutionary process.

Previous studies were largely focused on the properties of those galaxies located in the dense environments like cluster cores, highlighting mainly the effect of the subsequent evolutionary process like hierarchical merging, galaxy-galaxy interactions, and so on (Dressler 1980; Postman \& Geller 1984; Whitmore et al. 1993; Lewis et al. 2002; Tanaka et al. 2004). Yet, significant as its effect may be, the evolutionary process by itself is incapable of explaining the whole picture. There are many aspects of the environmental variations of galaxy properties that cannot be explained by the effect of the evolutionary process. For example, the analysis of SDSS data has revealed recently that the large void galaxies have very young stellar populations and quite high specific star formation rate (Rojas et al. 2005). Since the rates of galaxy merging and interaction in voids are quite low, it is unlikely due to the effect of the evolutionary process. Although it was claimed by Vogeley et al. (2004) that it is consistent with the predictions of the semi-analytic model of the galaxy formation (Benson et al. 2003), it has not been understood yet what caused the galaxies in voids to have such properties.

What is generally suspected is that the initial cosmological conditions should be at least partly responsible for establishing the environment-variation of galaxy properties (e.g., Tanaka et al. 2004). However, it is hard to investigate theoretically the effect of the initial cosmological conditions as it is still unknown under what initial conditions the formation of galaxies occurs. A conventional idea is that the galaxies form at the local peaks of the initial density field. If this is really the case, then it is unlikely that the initial conditions establish any strong dependence on the environments since the gravitational collapse of the local density peaks must depend mostly on the mass enclosed rather than the environments around the peaks (Sheth \& Tormen 2004).

However, there is another effect other than the local gravity that is responsible the formation of galaxies. At the initial stages, proto-galaxies interact with the surrounding matter, which originates the angular momentum. If the efficiency of the angular momentum generation by the external tidal field depends on the density of the environment, then the 
properties of galaxies like the morphology and the specific star formation rate may end up with having the environmental dependence.

In this Letter, we construct a new theoretical model for the efficiency of the generation of the galaxy angular momentum as a function of the density of the environment, and show qualitatively how the environmental dependence of the galaxy properties should be at least partly established by the initial tidal condition.

\section{THE INITIAL TIDAL CONDITION}

In the tidal-torque scenario (Hoyle 1949; Peebles 1969), a proto-galaxy acquires the angular momentum $\mathbf{L}=\left(L_{i}\right)$ through tidal interaction with the surrounding matter. The linear perturbation theory enables us to compute the angular momentum of a proto-galaxy in terms of Lagrangian quantities. According to this theory, if the shape of a proto-galaxy is not perfectly spherical, its angular momentum can be expressed as (Doroshkevich 1970; White 1984; Catelan \& Theuns 1996)

$$
L_{i} \propto \epsilon_{i j k} T_{j l} I_{l k} .
$$

This is the first-order approximation of the proto-galaxy angular momentum. Here, $\epsilon_{i j k}$ is an alternating symbol, and $\mathbf{I}=\left(I_{i j}\right)$ and $\mathbf{T}=\left(T_{i j}\right)$ are the inertia momentum tensor of the proto-galaxy and the tidal shear tensor of the surrounding matter, respectively. The definitions of the two tensors are given, respectively as

$$
I_{i j} \equiv \int_{V_{L}} q_{i} q_{j} d^{3} q,\left.\quad T_{i j} \equiv \partial_{i} \partial_{j} \phi\right|_{\mathbf{q}_{c m}},
$$

where $V_{L}$ is the Lagrangian volume of a proto-galaxy, $\mathbf{q}=\left(q_{i}\right)$ is the Lagrangian position vector in the center-of-mass frame, $\mathbf{q}_{c m}$ is the Lagrangian center-of-mass of the proto-galaxy, and $\phi$ is the initial velocity potential, related to the linear density contrast $\delta \equiv(\rho-\bar{\rho}) / \bar{\rho}$ as $\delta=\nabla_{\mathbf{q}}^{2} \phi(\mathbf{q})(\bar{\rho}$ : the mean density of the background universe).

Since we consider the generation of the angular momentum on the proto-galactic mass scale, the density field $\delta$ is supposed to be averaged over the proto-galactic mass scale $M$. In other words, the linear density $\delta$ is not the actual one but the smoothed one by convolution with a filter on the proto-galaxy mass scale. Accordingly, the tidal tensor $\mathbf{T}$ in equation (2) is a smoothed version of the actual tidal field on the mass scale $M$. In order to show explicitly that the tidal tensor in equation (2) is smoothed on the proto-galaxy mass scale $M$, we use the superscripted notation, $\mathbf{T}^{M}$, from here on. The same notation rule applies to

the inertia tensor $\mathbf{I}^{M}$ since it represents the geometrical shape of the proto-galaxy, defined 
on the same mass scale $M$. Thus, equations (1)-(2) imply that the proto-galaxy angular momentum is generated by the tidal field smoothed on the same mass scale as the inertia tensor.

Adopting a top-hat spherical filter whose functional form in the Fourier space is given as

$$
W(k R)=\frac{k R \sin (k R)-\cos (k R)}{k R},
$$

one can relate the proto-galactic mass $M$ to the filtering radius $R$ as

$$
M(R)=\frac{4 \pi}{3} \bar{\rho}(1+\delta) R^{3} .
$$

Here, the filtering radius $R$ represents the effective spherical radius (i.e., the linear size) of the proto-galaxy $R \propto V_{L}^{1 / 3}$.

The tidal interaction of the proto-galaxy with the surrounding materials continues till the moment of the turn-around when $\delta \sim 1$. After the moment of the turn-around, the tidal influence from the surrounding matter effectively terminates, bringing the growth of the angular momentum to a halt. Thus, it is at the moment of the turn-around when the tidal effect of the surrounding matter is most influential. Although the nonlinear effect like galaxy-galaxy interaction might modify the galaxy angular momentum, it have been verified both numerically and observationally that the angular momentum of galaxies at present epoch still keeps quite well the initial memory of the surrounding matter distribution at the moment of the turn-around (Navarro et. al. 2004; Trujillo et. al. 2005).

Now, let us express equation (1) in the principal axis frame of the tidal tensor $\mathbf{T}$ :

$$
L_{1} \propto\left(\lambda_{1}^{M}-\lambda_{2}^{M}\right) I_{12}^{M}, \quad L_{2} \propto\left(\lambda_{2}^{M}-\lambda_{3}^{M}\right) I_{23}^{M}, \quad L_{3} \propto\left(\lambda_{3}^{M}-\lambda_{1}^{M}\right) I_{31}^{M},
$$

where $\lambda_{1}^{M}, \lambda_{2}^{M}, \lambda_{3}^{M}$ are the three eigenvalues of the tidal tensor $\mathbf{T}^{M}$ in a decreasing order, and $I_{12}^{M}, I_{23}^{M}, I_{31}^{M}$ are the three off-diagonal components of the inertia tensor $\mathbf{I}^{M}$ in the principal axis frame of the tidal tensor $\mathbf{T}^{M}$.

Equation (5) implies that only in case the principal axes of the inertia tensor $\mathbf{I}^{M}$ are not aligned with that of the tidal tensor $\mathbf{T}^{M}$, the angular momentum can be generated at first order. It also implies that the degree of the misalignment between the principal axes of $\mathbf{T}^{M}$ and $\mathbf{I}^{M}$ is directly proportional to the magnitude of the angular momentum. Recent N-body simulations demonstrated that the principal axes of the inertia tensor $\mathbf{I}^{M}$ are almost perfectly aligned with that of the tidal tensor $\mathbf{T}^{M}$, if the two tensors are smoothed at the same radius (Lee \& Pen 2000; Porciani et al. 2002).

This empirical finding may be understood theoretically. In the Zel'dovich approximation 
(Zeldovich 1970), a proto-galaxy forms at the moment of shell-crossing when the principal axes of the tidal tensors are perfectly aligned with that of the inertia tensors.

But, it is still possible for the galaxy angular momentum to be generated at first order. Although the tidal interaction is a local effect, the external tidal field has a large-scale coherence (Bond, Kofman, \& Pogosyan 1996). Thus, the tidal tensor smoothed at larger scales may be misaligned with the inertia tensor, and thus it can generate the galaxy angular momentum at first order.

Let $\delta_{1}$ and $R_{1}$ be the linear density contrast and the effective spherical radius of the proto-galaxy of mass $M$. To consider the effect of the external tidal field smoothed at larger scales, we assume that the proto-galaxy is located in the environment whose linear density contrast and the scale radius are given as $\delta_{2}<\delta_{1}$ and $R_{2}>R_{1}$, respectively, satisfying a condition that the larger scale radius $R_{2}$ should enclose the same amount of mass, $M$. This condition guarantees that even though the external field is smoothed at larger scale $R_{2}$, it represents a local tidal effect acting on the proto-galactic mass $M$. Using the condition $M\left(R_{1}\right)=M\left(R_{2}\right)$, we have

$$
R_{2}=R_{1}\left(\frac{1+\delta_{1}}{1+\delta_{2}}\right)^{1 / 3}
$$

Since the values of $R_{1}$ and $\delta_{1}$ are fixed, equation (6) implies that the lower the linear density $\delta_{2}$ is, the larger is the scale radius $R_{2}$.

Now that the tidal tensor $\mathbf{T}^{M}$ is smoothed at larger scale $R_{2}$, it is no longer perfectly aligned with the inertia tensor $\mathbf{I}^{M}$ smoothed at scale $R_{1}$. The misalignment between $\mathbf{T}^{M\left(R_{2}\right)}$ and $\mathbf{I}^{M\left(R_{1}\right)}$ is expected to increase as the difference between $R_{1}$ and $R_{2}$ increases. To quantify the misalignment between $\mathbf{T}^{M\left(R_{2}\right)}$ and $\mathbf{I}^{M\left(R_{1}\right)}$, we first rescale both the tidal and the inertia tensors to have unit magnitudes and become traceless as

$$
\hat{\mathbf{T}}^{M\left(R_{2}\right)} \equiv \frac{\tilde{\mathbf{T}}^{M\left(R_{2}\right)}}{\left|\tilde{\mathbf{T}}^{M\left(R_{2}\right)}\right|}, \quad \hat{\mathbf{I}}^{M\left(R_{1}\right)} \equiv \frac{\tilde{\mathbf{I}}^{M\left(R_{1}\right)}}{\left|\tilde{\mathbf{I}}^{M\left(R_{1}\right)}\right|},
$$

where $\left|\tilde{\mathbf{T}}^{M\left(R_{2}\right)}\right| \equiv\left[\tilde{T}_{i j}^{M\left(R_{2}\right)} \tilde{T}_{i j}^{M\left(R_{2}\right)}\right]^{1 / 2}$ and $\left|\tilde{\mathbf{I}}^{M\left(R_{1}\right)}\right| \equiv\left[\tilde{I}_{i j}^{M\left(R_{1}\right)} \tilde{I}_{i j}^{M\left(R_{1}\right)}\right]^{1 / 2}$, with $\tilde{\mathbf{T}} \equiv \mathbf{T}-\operatorname{Tr}(\mathbf{T}) / 3$ and $\tilde{\mathbf{I}} \equiv \mathbf{I}-\operatorname{Tr}(\mathbf{I}) / 3$.

By equation (1), the square of the magnitude of the proto-galaxy specific (per mass) angular momentum is proportional to

$$
\left\langle L^{2}\right\rangle \propto \epsilon_{i a b} \epsilon_{i u v}\left\langle\hat{T}_{a c}^{M\left(R_{2}\right)} \hat{T}_{c b}^{M\left(R_{2}\right)} \hat{I}_{u w}^{M\left(R_{1}\right)} \hat{I}_{w v}^{M\left(R_{1}\right)}\right\rangle .
$$

Since the principal axes of the inertia and the tidal tensors smoothed at the same filtering radius are almost perfectly correlated, we make an approximation $\hat{\mathbf{I}}^{M\left(R_{1}\right)} \approx \hat{\mathbf{T}}^{M\left(R_{1}\right)}$ in 
equation (8), and we define $\beta$ as

$$
\beta \equiv\left[\epsilon_{i a b} \epsilon_{i u v}\left\langle\hat{T}_{a c}^{M\left(R_{2}\right)} \hat{T}_{c b}^{M\left(R_{2}\right)} \hat{T}_{u w}^{M\left(R_{1}\right)} \hat{T}_{w v}^{M\left(R_{1}\right)}\right\rangle\right]^{1 / 2} .
$$

The value of $\beta$ basically represents the efficiency of the generation of the galaxy angular momentum at first order. It increases as the degree of the misalignment between the principal axes of the tidal tensor $\mathbf{T}^{M\left(R_{2}\right)}$ and $\mathbf{T}^{M\left(R_{1}\right)}$ increases. Thus, it is expected that it increases as the density of the environment decreases according to equation (6).

To see this explicitly, we evaluate the value of $\beta$, using the following approximation whose validity was justified by the Monte-Carlo simulation (Lee \& Pen 2001):

$$
\left\langle\hat{T}_{a c}^{M\left(R_{2}\right)} \hat{T}_{c b}^{M\left(R_{2}\right)} \hat{T}_{u w}^{M\left(R_{1}\right)} \hat{T}_{w v}^{M\left(R_{1}\right)}\right\rangle \approx \frac{\left\langle\tilde{T}_{a c}^{M\left(R_{2}\right)} \tilde{T}_{c b}^{M\left(R_{2}\right)} \tilde{T}_{u w}^{M\left(R_{1}\right)} \tilde{T}_{w v}^{M\left(R_{1}\right)}\right\rangle}{\left|\tilde{\mathbf{T}}^{M\left(R_{2}\right)}\right|^{2}\left|\tilde{\mathbf{T}}^{M\left(R_{1}\right)}\right|^{2}} .
$$

By the Wick theorem, $\left\langle\tilde{T}_{a c}^{M\left(R_{2}\right)} \tilde{T}_{c b}^{M\left(R_{2}\right)} \tilde{T}_{u w}^{M\left(R_{1}\right)} \tilde{T}_{w v}^{M\left(R_{1}\right)}\right\rangle$, equation (10) is written as

$$
\begin{aligned}
\left\langle\tilde{T}_{a c}^{M\left(R_{2}\right)} \tilde{T}_{c b}^{M\left(R_{2}\right)} \tilde{T}_{u w}^{M\left(R_{1}\right)} \tilde{T}_{w v}^{M\left(R_{1}\right)}\right\rangle & =\left\langle\tilde{T}_{a c}^{M\left(R_{2}\right)} \tilde{T}_{c b}^{M\left(R_{2}\right)}\right\rangle\left\langle\tilde{T}_{u w}^{M\left(R_{1}\right)} \tilde{T}_{w v}^{M\left(R_{1}\right)}\right\rangle \\
& +\left\langle\tilde{T}_{a c}^{M\left(R_{2}\right)} \tilde{T}_{w v}^{M\left(R_{1}\right)}\right\rangle\left\langle\tilde{T}_{u w}^{M\left(R_{1}\right)} \tilde{T}_{c b}^{M\left(R_{2}\right)}\right\rangle \\
& +\left\langle\tilde{T}_{a c}^{M\left(R_{2}\right)} \tilde{T}_{u w}^{M\left(R_{1}\right)}\right\rangle\left\langle\tilde{T}_{w v}^{M\left(R_{1}\right)} \tilde{T}_{c b}^{M\left(R_{2}\right)}\right\rangle .
\end{aligned}
$$

The correlations between the components of the tidal tensors can be written as

$$
\left\langle T_{i j}^{M\left(R_{1}\right)} T_{k l}^{M\left(R_{2}\right)}\right\rangle=\partial_{i} \partial_{j} \partial_{k} \partial_{l} \nabla^{-4} \int P(k) W_{T H}\left(k ; R_{1}\right) W_{T H}\left(k ; R_{2}\right) d^{3} k .
$$

Through equations (9)-(12), it is straightforward to derive

$$
\beta=\left(1-\frac{\sigma_{c}^{4}}{\sigma_{1}^{2} \sigma_{2}^{2}}\right)^{1 / 2}
$$

where the three mass variances $\sigma_{1}^{2}, \sigma_{2}^{2}, \sigma_{c}^{2}$ are defined as

$$
\begin{aligned}
\sigma_{1}^{2} & =\frac{1}{2 \pi^{2}} \int P(k) W^{2}\left(k R_{1}\right) k^{2} d k, \\
\sigma_{2}^{2} & =\frac{1}{2 \pi^{2}} \int P(k) W^{2}\left(k R_{2}\right) k^{2} d k, \\
\sigma_{c}^{2} & =\frac{1}{2 \pi^{2}} \int P(k) W\left(k R_{1}\right) W\left(k R_{2}\right) k^{2} d k,
\end{aligned}
$$

with the linear power spectrum $P(k)$.

Figure 1 plots $\beta$ as a function of the linear density contrast of the environment $\delta_{2}$ through equations (6)-(16). For $P(k)$ in equations (14)-(15), we use the approximation given by 
Bardeen et al. (1986) for the concordance cosmology with $\Omega_{m}=0.3, \Omega_{\Lambda}=0.7, h=0.7, \Omega_{b}=$ 0.044 , and $\sigma_{8}=0.9$. For this plot, we use the typical galaxy mass scale $M=10^{12} h^{-1} M_{\odot}$. As seen clearly, $\beta$ decreases monotonically as $\delta_{2}$ increases, reducing to zero as the value of

$\delta_{2}$ approaches $\delta_{1}$. This result shows how the generation of the galaxy angular momentum depend on the environment: high-angular momentum in the low-density region $\left(\delta_{2}<<\delta_{1}\right)$ and low-angular momentum in the high-density region $\left(\delta_{2} \sim \delta_{1}\right)$. It provides a theoretical explanation to the recent numerical finding that the spin parameters of void galaxies are higher than those of cluster galaxies (Avila-Reese et al. 2005).

\section{DISCUSSION AND CONCLUSION}

In the cold dark matter (CDM) paradigm, the basic mechanism for the star formation in galaxies is described as follows. The hot gas falls into the gravitational potential well of the galactic dark halo, and goes through radiative cooling. A cloud of the cool gas in the halo potential well fragments into many clumps, and those clumps can further collapse via self-gravity to form stars. The angular momentum of a galactic dark halo hinders the formation of stars in it: The high angular momentum causes high rotational speed of the gas clumps, which slows down the collapse of clumps into stars.

In $\S 2$, it is shown that the galaxies in the low-density environment will end up with having high angular momentum. Consequently, it will take long time before stars form in the galaxies located in the low-density environment. For example, if a galaxy is located in the lowest-density environment like a void, then stars in the void galaxy must form only very recently. In other words, the void galaxies are predicted to have young stellar populations and show higher specific star formation rate at present epoch. One should note here that it is true only for those void galaxies whose sizes are large enough to hold gas. If a void galaxy is small, then the UV-background will photo-evaporate all gas in the galaxy, suppressing any star-formation activity (e.g., Susa \& Umemura 2004; Hoeft et al. 2005).

This theoretical insight agrees with the recent observational report that the large void galaxies at fixed luminosity and surface brightness profile are disk-like, having bluer colors and higher specific star formation rate than the wall galaxies, which was not explained by the previous morphology-density relation (Vogeley et al. 2004).

The properties of galaxies located in the high-density environment like galaxy clusters may be explained in the same line. A low angular momentum is expected for a proto-galaxy in a cluster due to the strong alignment between the inertia and the tidal tensors in the high-density. Thus, it will be quite faster to form stars in the cluster galaxies, because there 
is no hindrance caused by the galaxy angular momentum. However, for the cluster galaxies, it is expected that the evolutionary process like merging and interaction must play more significant role in establishing the environmental dependence.

As a final conclusion, we have provided a new insight into the puzzle of galaxy formation by showing that the environmental dependence of galaxy properties has an aspect of destiny, being caused by the initial cosmological conditions.

J.L. acknowledges stimulating discussion with W. T. Kim. This work is supported by the research grant No. R01-2005-000-10610-0 from the Basic Research Program of the Korea Science and Engineering Foundation. 


\section{REFERENCES}

Avila-Reese, V., Colin, P., Gottlober, S., Firmani, C., \& Firmani, C. 2005, ApJ, 634, 51

Abbas, U., \& Sheth, R. K. 2005, MNRAS, 364, 1327

Balogh, M. et al. 2004, MNRAS, 348, 1355

Bardeen, J. M., Bond, J. R., Kaiser, N., \& Szalay, A. S. 1986, ApJ, 304, 15

Benson, A. J., Hoyle, F., Torres, F., \& Vogeley, M. S. 2003, MNRAS, 340, 160

Bond, J., R., Kofman, L., \& Pogosyan, D. 1996, Nature, 380, 603

Catelan, P. \& Theuns, T. 1996, MNRAS, 282, 436

Colless et al. 2001, MNRAS, 328, 1039

Doroshkevich, A. G. 1970, astrofizika, 6, 581

Dressler, A. 1980, ApJ, 236, 351

Gomez, P. et al. 2003, ApJ, 584, 210

Goto, et al. 2003, MNRAS, 346, 601

Hao, L., Strauss, M. A., Rojas, R. R., \& Vogeley, M. S. 2004,

Hoeft, M., Yepes, G., Gottloeber, S., \& Springel, V. 2005, preprint [astro-ph/0501304]

Hoyle, F. 1949, in Problems of Cosmical Aerodynamics, ed. J. M. Burgers \& H. C. van de Hulst (Dayton: Central Air Documents Office), 195

Peebles, P. J. E. 1969, ApJ, 155, 393

Lee, J. \& Pen, U. L. 2000, ApJ, 532, L5

Lee, J. \& Pen, U. L. 2001, ApJ, 555, 106

Lewis, I., et al. 2002, MNRAS, 334, 673

Navarro, J.F., Abadi, M.G., \& Steinmetz, M. 2004, ApJ, 613, L41

Porciani, C., Dekel, A., \& Hoffman, Y. 2002, MNRAS, 332, 339

Postman, M., \& Geller, M. J., 1984, ApJ, 281, 95 
Rines, K., Geller, M. J., Kurtz, M. J., Diaferio, A. 2005, AJ, 130, 1482

Rojas, R. R., Vogeley, M. S., Hoyle, F., \& Brinkmann, J. 2005, 624, 571

Sheth, R. K., \& Tormen, G. 2004, MNRAS, 349, 1464

Strauss, et al. 2002, ApJ, 124, 1810

Susa, H., \& Umemura, M. 2004, 610, L5

Tanaka, M., Goto, T., Okamura, S., Shimasaku, K., \& Brinkman, J. 2004, AJ, 128, 2677

Trujillo, I., Carretero, C., \& Patiri, S. 2005, ApJin press

Vogeley, M. S. et al. 2004, Outskirts of Galaxy Clusters, Proceedings IAU Colloquium No. 195

White, S. D. M. 1984, ApJ, 286, 38

Whitmore, B. C., Gilmore, D. M., \& Jones, C. 1993, ApJ, 407, 489

Zel'dovich, Y. B. 1970, A\& A, 5, 84 


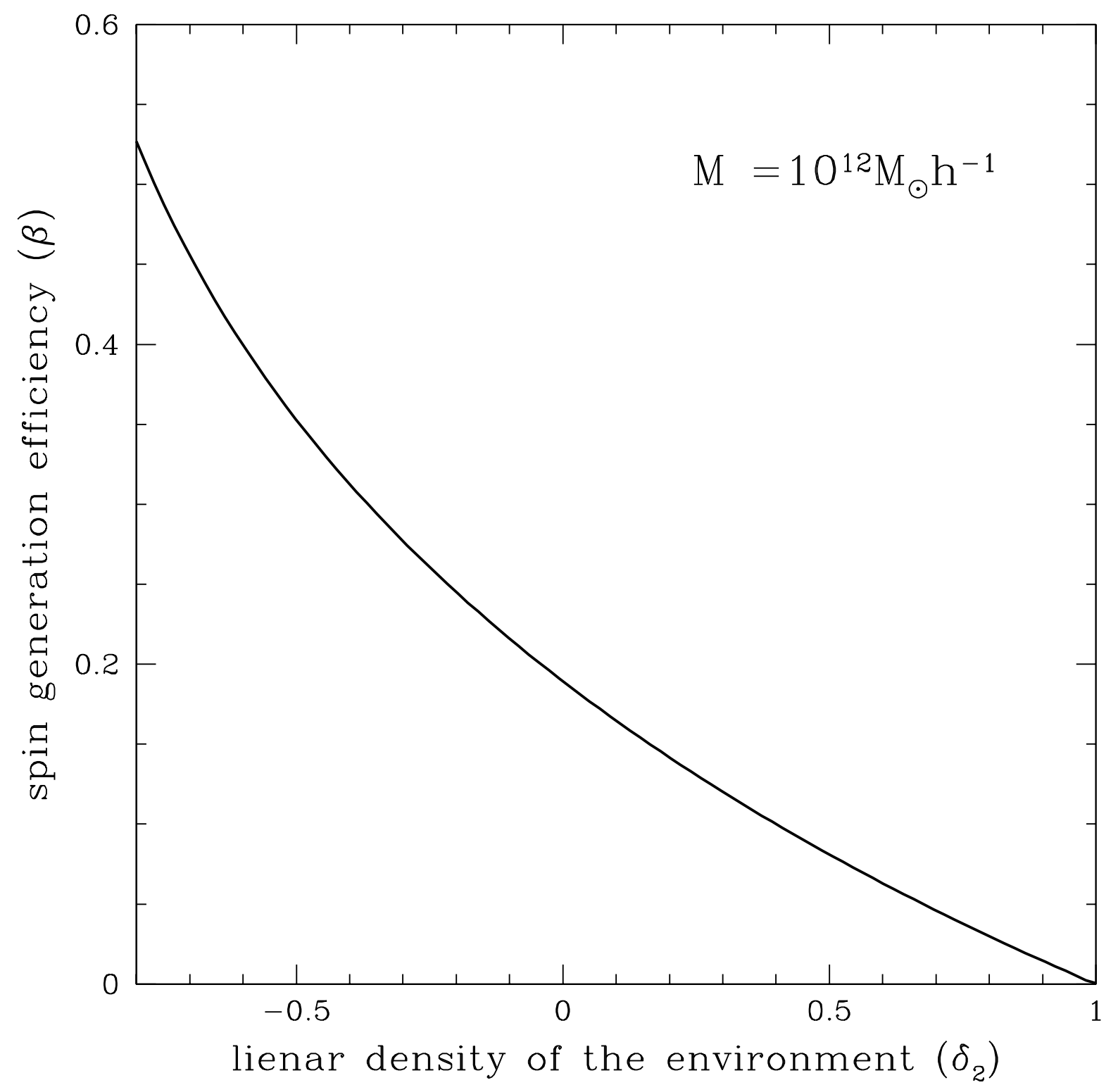

Fig. 1.- Spin-generation efficiency of a galactic dark halo with mass $10^{12} h^{-1} M_{\odot}$ as a function of the linear density contrast of the environment where the galaxy is located. 